\title{
IVIG: The Heart Savior in Covid 19 era
}

Rima Chaddad MD ${ }^{1 *}$, Matina Hamadeh $\mathrm{MD}^{2}$, Amena Khatoun ${ }^{2}$, Zouheir Kreidly $\mathrm{MD}^{3}$, Claudette Najjar MD ${ }^{4}$, Pierre Nassar MD ${ }^{5}$, Malek Moussa MD

${ }^{1}$ Cardiology fellow, Lebanese University

${ }^{2}$ Infectious disease fellow, Lebanese University

${ }^{3}$ Infectious disease Specialist, Beirut Cardiac Institute

${ }^{4}$ Cardiologist, Echocardiographist, Beirut Cardiac Institute

${ }^{5}$ Cardiologist, Cardiac MRI and Imaging Specialist, Beirut Cardiac Institute

${ }^{6}$ Cardiologist, Cardiac Care Unit, Beirut Cardiac Institute

*Corresponding author: Rima Chaddad, Cardiology fellow, Lebanese University.

Received Date: June 25, 2021; Accepted Date: July 06, 2021; Published Date: July 09, 2021

Citation: R Chaddad, M Hamadeh, A Khatoun, Z Kreidly, C Najjar, et al. (2021) IVIG : The Heart Savior in Covid 19 era. Cardiology Research and Reports. 3(3): Doi:10.31579/2692-9759/026

Copyright: () 2021 Rima Chaddad. This is an open-access article distributed under the terms of the Creative Commons Attribution License, which permits unrestricted use, distribution, and reproduction in any medium, provided the original author and source are credited.

\begin{abstract}
Coronavirus disease 2019 (COVID-19) is defined as illness caused by a novel coronavirus now called severe acute respiratory syndrome coronavirus 2 (SARS-CoV-2; formerly called 2019-nCoV), which was first identified as an outbreak in Wuhan City, Hubei Province, China [1].

Cases of multisystem inflammatory syndrome after Covid - 19 infection have been extensively documented in children. CDC reported 27 cases of multisystem inflammatory syndrome in adults (MIS-A) in direct reports through October 2020, as well as various case series and published case reports [2].

Cytokine storm, as a trigger of the progression of HF in patients with COVID-19, has become a novel focus to explore therapies for target populations. ${ }^{[3]}$

In this article, we briefly present a case of a Covid-19 infection associated myocarditis complicated by acute heart failure successfully treated with IVIG.
\end{abstract}

Keywords: COVID-19; MIS-A, myocarditis; IVIG

\section{Introduction}

Severe Acute Respiratory Syndrome-Coronavirus-2 (SARS-CoV-2) is an unmatched challenge for the healthcare community across the world. Respiratory involvement is the main clinical manifestation of COVID-19, ranging from mild flu-like illness to severe pneumonia, and potentially lethal acute respiratory distress syndrome.

A multisystem inflammatory syndrome occurring several weeks after SARS-CoV-2 infection and that can include severe acute heart failure has been reported in children (MIS-C). In adults with acute severe heart failure, we have identified a similar syndrome (MIS-A) [4].

Following infection, adults with acute COVID-19 often experience fever accompanied by respiratory symptoms (eg, cough, shortness of breath). A small proportion of patients progress to a later stage of COVID-19, at times referred to as the cytokine storm. This late stage can include coagulopathy and multiorgan dysfunction including myocardial dysfunction in the setting of severe inflammation [3].
This report is an important addition to descriptions of MIS-A.

\section{Case presentation}

A 27 year-old man presented to the emergency room for evaluation of progressively worsening dyspnea, fever, myalgia and diarrhea over 1 week. His past medical history was relevant for Covid -19 infection 2 months ago. He was admitted in another hospital for 3 days, where he was diagnosed as MIS-A and treated with steroids and Tocilizumab $800 \mathrm{mg}$ iv single dose the second day of admission (Interleukin 6 level unavailable).

Myalgia and diarrhea were significantly improved, but referred to our cardiac center the third day because of his worsening dyspnea and orthopnea and acute onset systolic heart failure (TTE showed depressed LV function).

Upon presentation to our Emergency Department, patient was severely dyspneic with rapid shallow breathing . 
Vitals showed a temperature of 38.5 degree $\mathrm{C}$, an oxygen saturation of 80 $\%$ requiring non rebreather mask, a heart rate of $120 \mathrm{bpm}$ and a blood pressure of $100 / 70 \mathrm{mmHg}$.
Physical exam revealed diminished breath sounds at lung bases with crackles.

ECG showed sinus tachycardia. (Figure .1)

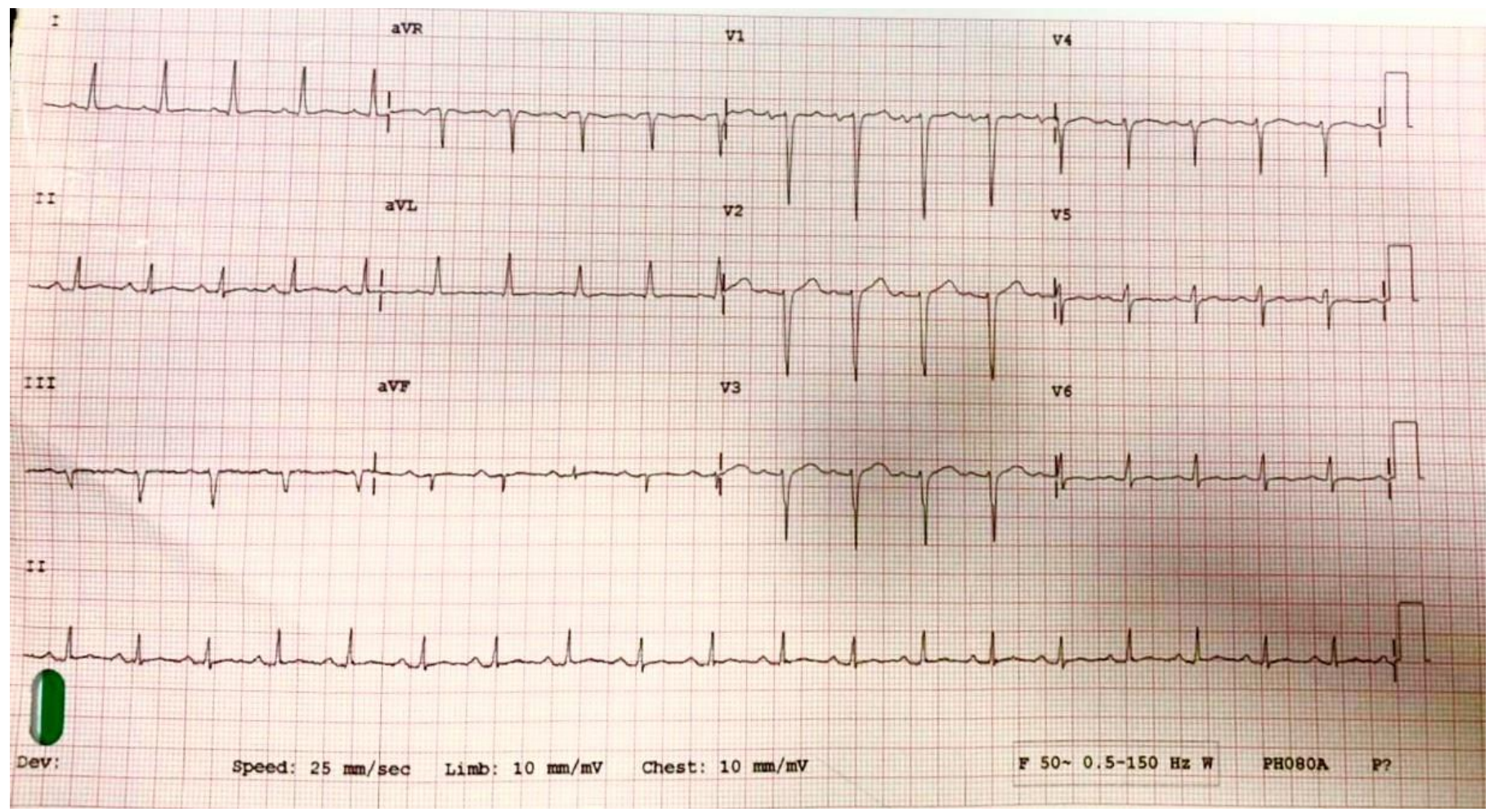

Figure 1: ECG: Sinus Tachycardia.

Chest X-ray demonstrated hilar congestion. (Figure.2)

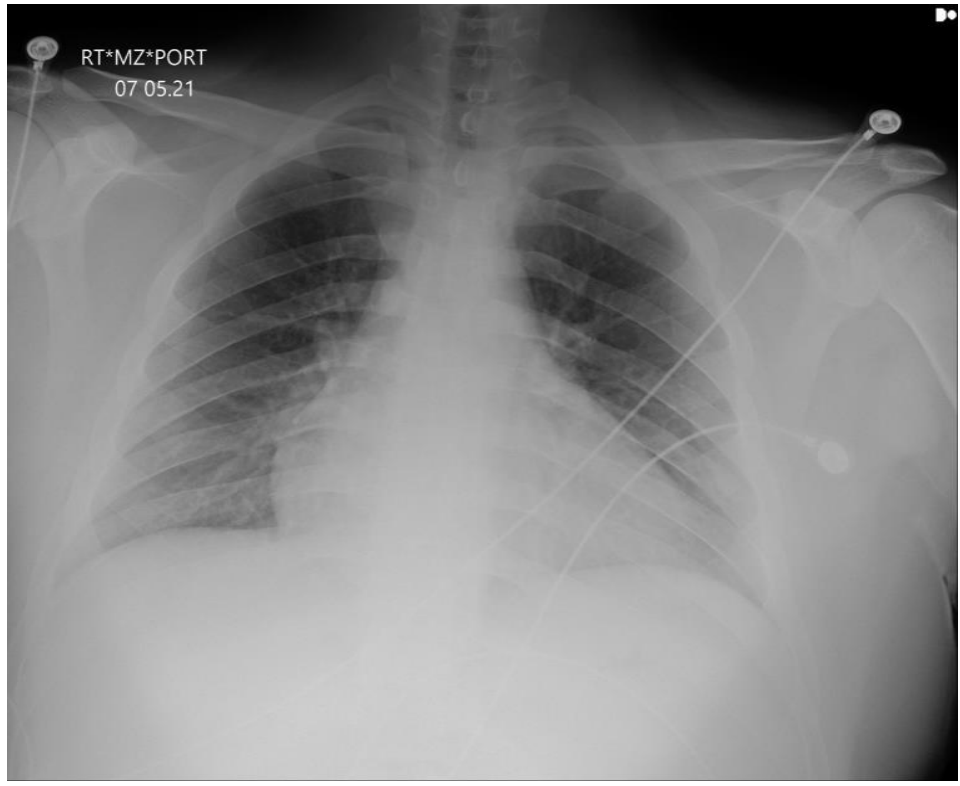

Figure 2: CXR: Cardiomegaly with hilar congestion.

Initial laboratory investigations showed an elevated inflammatory markers, and cardiac biomarkers with multiorgan damage. (Table 1)

PCR Covid -19 was negative with an IgG Covid-19 level of 10.
A chest computed tomography pulmonary angiography (CTPA) demonstrated mild bilateral ground-glass opacities suggesting residual Covid -19 infection and possible infiltrates with no pulmonary embolism noted (Figure.3). 


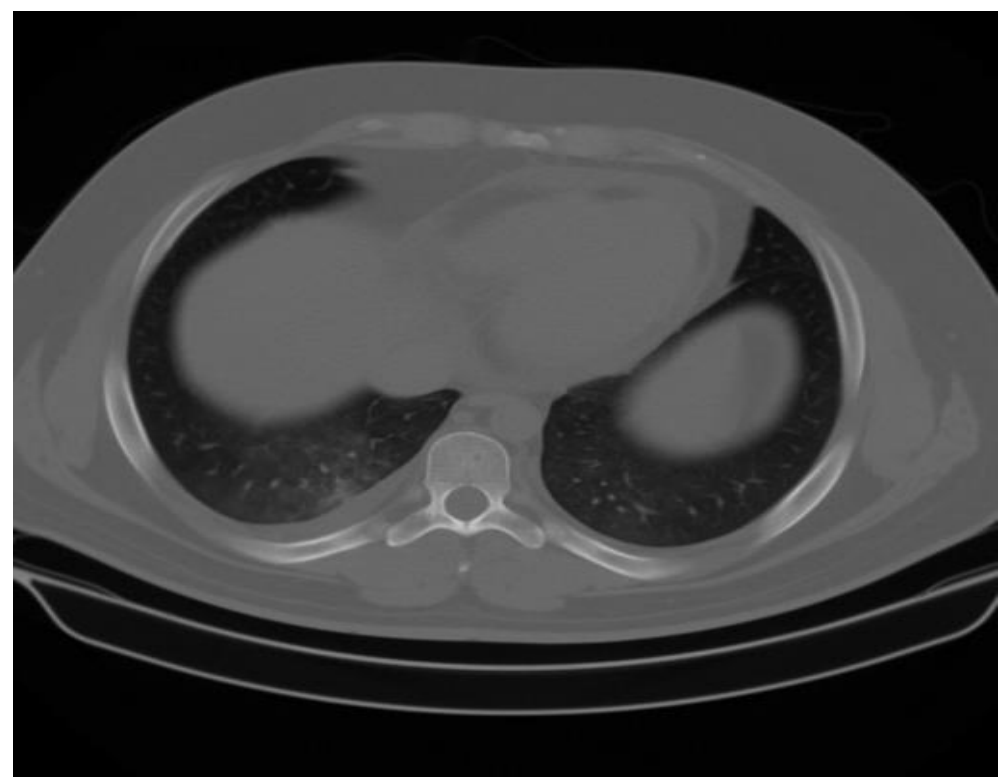

Figure 3: CTA Chest: Basilar ground glass opacities, pericardial effusion.

Transthoracic echocardiogram (TTE) showed severe globally depressed left ventricular systolic function [left ventricular ejection fraction (LVEF) of $28 \%$ ] and mild pericardial effusion (Figure.4).

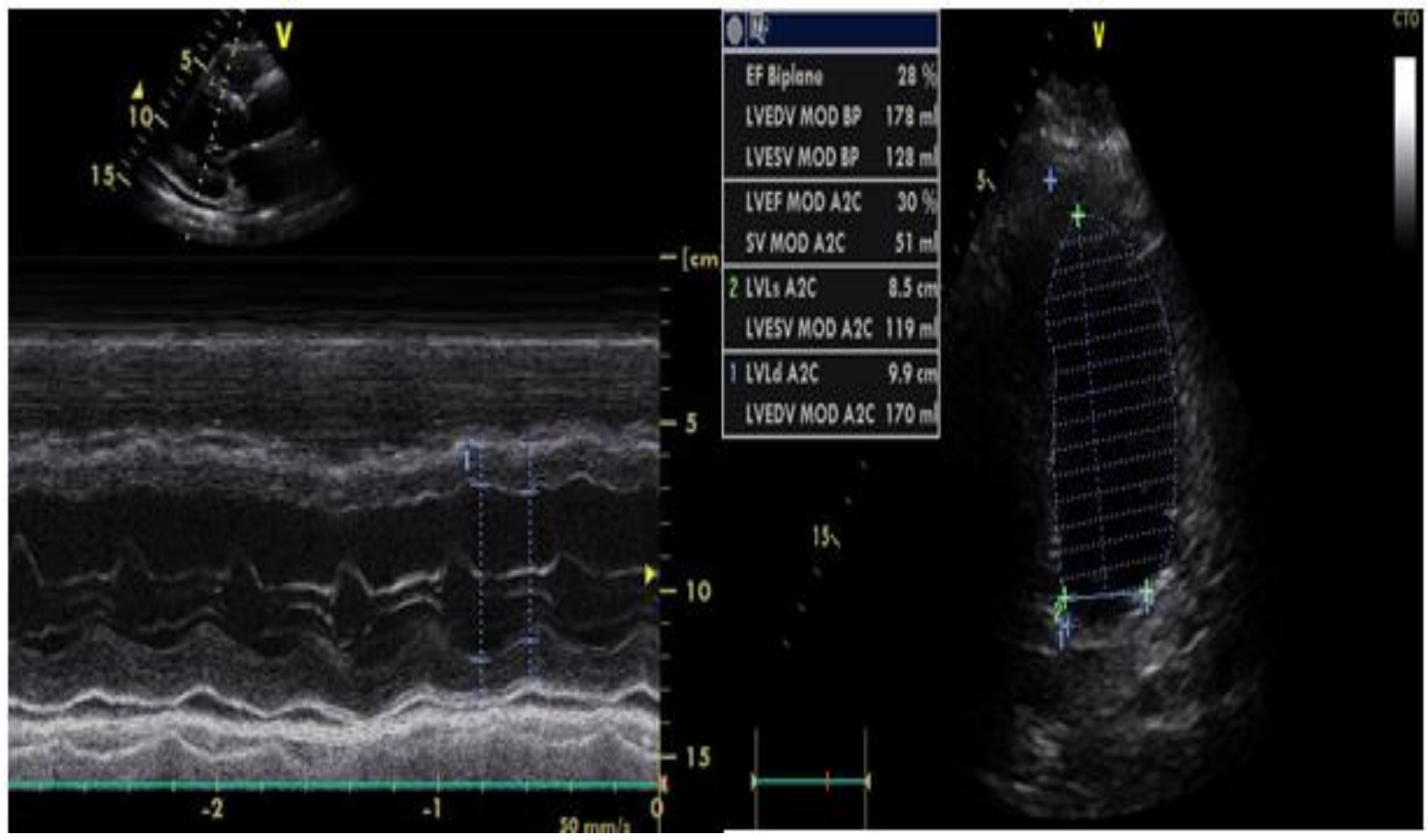

Figure 4: Echocardiography:M-Mode and Simpson biplane showed Depressed LV systolic function:28\%.

The differential diagnosis of new-onset acute left ventricular heart failure included sepsis-induced cardiomyopathy, Takotsubo syndrome, viral lymphocytic myocarditis in the setting of multisystem inflammatory syndrome.
Cardiac MRI showed probable acute myocarditis mainly involving the lateral wall with mild pericardial effusion (Figure .5). 


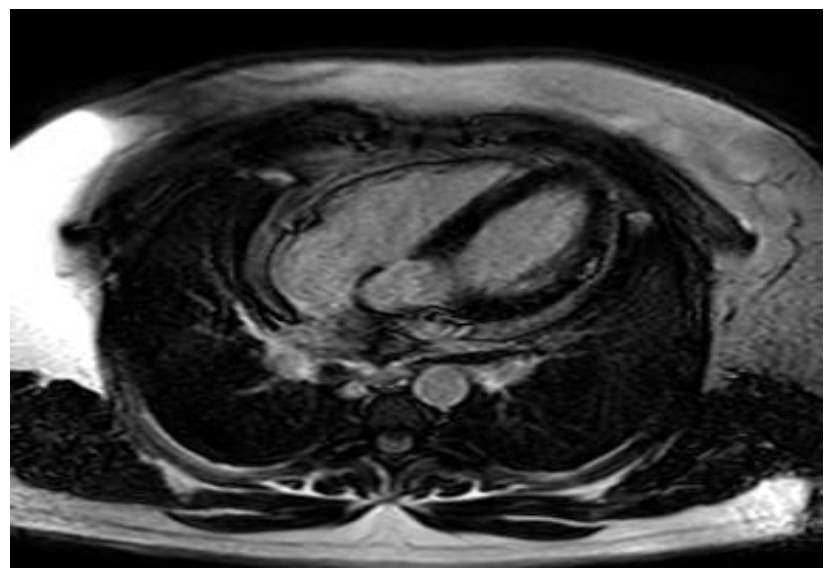

Figure 5: Cardiac MRI: Subepicardic LGE uptake in the myocardium at the level of the lateral wall.

The patient received Immunoglobulins (IVIG) $40 \mathrm{~g}$ for 3 days for suspicion of viral myocarditis and steroids (solumedrol $40 \mathrm{mg}$ iv $\mathrm{q} 8 \mathrm{~h}$ ) for the cytokine storm in addition to colchicine $0.5 \mathrm{mg}$ daily and broad spectrum antibiotics.
Patient also started on heart failure treatment on the second day.

Within $48 \mathrm{~h}$ of the above interventions, the patient experienced significant clinical improvement with down-trending inflammatory markers and less oxygen requirement. (Table 1)

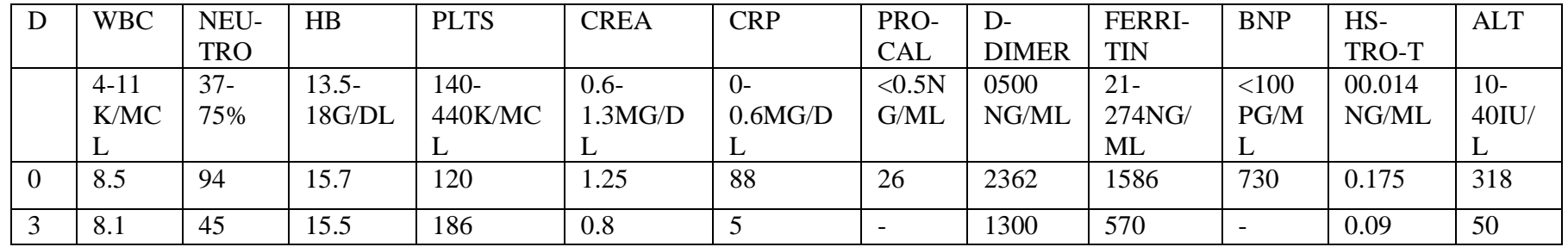

Continuous cardiac monitoring did not reveal any significant dysrhythmia.

Repeated TTE $72 \mathrm{~h}$ after CCU admission demonstrated significant myocardial recovery, showing an LVEF of $65 \%$, and a small pericardial effusion.
Day 4 post admission, patient was transferred to regular floor and discharged the next day.

After one month, patient was significantly improved, free of symptoms, returned to his regular daily activities without any problems.Follow up TTE showed a recovered LV function with LVEF: 64\% (Figure .6).

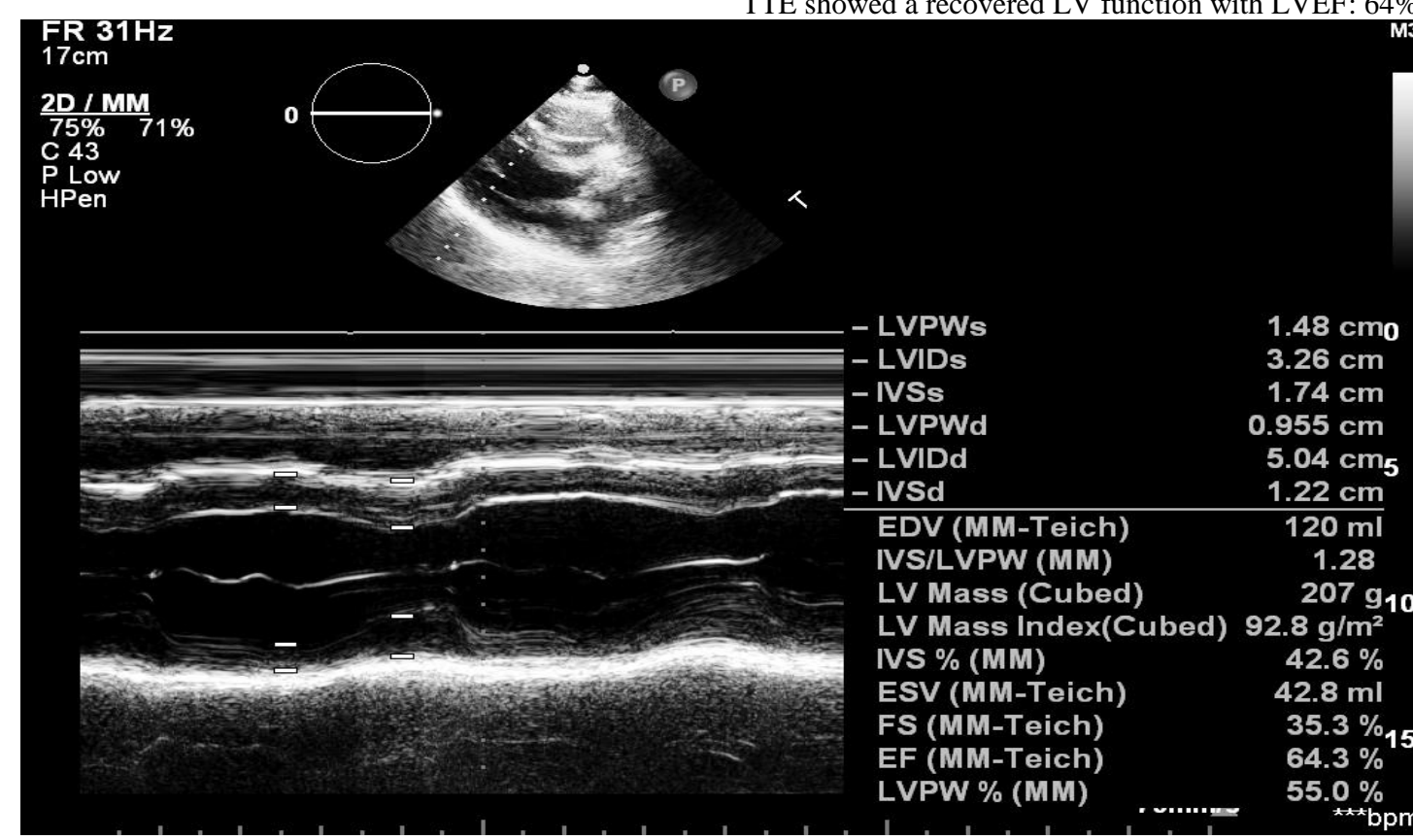

Figure 6: M-Mode showed LVEF: 64\%. 


\section{Discussion}

Findings indicate that adult patients of all ages with current or previous SARS-CoV-2 infection can develop a hyperinflammatory syndrome resembling MIS-C.

Current convention uses the CDC criteria which include: 1 . a severe illness requiring hospitalization in a person aged $\geq 21$ years; 2 . a positive test result for current or previous SARS-CoV2 infection (nucleic acid, antigen, or antibody) during admission or in the previous 12 weeks; 3 . Severe dysfunction of one or more extrapulmonary organ systems (e.g., hypotension or shock, cardiac dysfunction, arterial or venous thrombosis or thromboembolism, or acute liver injury); 4. Laboratory evidence of severe inflammation (e.g., elevated CRP, ferritin, D-dimer, or interleukin6 ); and 5. Absence of severe respiratory illness (to exclude patients in which inflammation and organ dysfunction might be attributable simply to tissue hypoxia) [1]

The pathophysiology of MIS in both children and adults is currently unknown. Eight of 27 (30\%) adults described in the literature and $45 \%$ of 440 children with MIS-C reported to CDC through July 29, 2020, had negative PCR and positive SARS-CoV-2 antibody test results, suggesting MIS-A and MIS-C might represent postinfectious processes.

SARS-CoV-2 has been identified in multiple organs including the heart, liver, brain, kidneys, and gastrointestinal tract [5]. Additional proposed mechanisms for extrapulmonary dysfunction in COVID-19 include endothelial damage and thromboinflammation, dysregulated immune responses, and dysregulation of the renin-angiotensin-aldosterone system [5].

In patients with COVID-19, dyspnea is typically experienced a median of 5-8 days and critical illness 10-12 days after onset of symptoms. In patients who reported typical COVID-19 symptoms before MIS-A onset, MIS-A was experienced approximately $2-5$ weeks later.

Antibody testing for SARS-CoV-2 might be needed to confirm previous COVID-19 infection in patients who do not have positive SARS-CoV-2 PCR or antigen test results. Further research is needed to understand the pathogenesis and long-term effects of this newly described condition. Ultimately, the recognition of MIS-A reinforces the need for prevention efforts to limit spread of SARS-CoV-2.

$\mathrm{HF}$ was deemed one of the most common complications occurring during the exacerbation of COVID-19, and these conditions were more likely to appear in patients with pre-existing cardiac diseases [6].

The pathophysiology of COVID-19-related myocarditis is thought to be a combination of direct viral injury and cardiac damage due to the host's immune response [7].

Intravenous immunoglobulin was used in three reported cases with variable outcomes. The hypothesis behind the use of

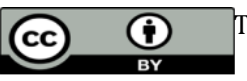

This work is licensed under Creative Commons Attribution 4.0 License

\section{To Submit Your Article Click Here: Submit Manuscript}

DOI: $10.31579 / 2692-9759 / 026$ intravenous immunoglobulin (IVIG) is that it may replace antibodies, augment viral clearance, and enhance clearance of inflammatory cytokines that contribute to myocyte destruction.

A meta-analysis published in 2019 comparing IVIG to corticosteroid for acute myocarditis

Showed that IVIG therapy improved mortality and recovery of left ventricular function [8].

In our case, early aggressive treatment of suspected myocarditis with IVIG therapy likely contributed to our patient's rapid recovery.

\section{Conclusion}

In conclusion, we report this rare case of myocarditis complicated by acute heart failure associated with SARS-CoV-2-induced cytokine storm that resolved with IVIG and supportive medications alone, and without the assistance of ECMO or MCS

A large randomized controlled trial is required to determine the efficacy of immunoglobulins in Covid-related myocarditis.

\section{References:}

1. World Health Organization

2. Bhaskar S, Sinha A, Banach M, Mittoo S, Weissert R, Kass JS et al. (2020) Cytokine Storm in COVID-19Immunopathological Mechanisms, Clinical Considerations, and Therapeutic Approaches: The REPROGRAM Consortium Position Paper.Front Immunol. 11:1648.

3. Hékimian G, Kerneis M, Zeitouni M, et al: (2020) COVID-19 acutemyocarditis and multisystem inflammatory syndrome in adult intensive and cardiac care units.

4. Datta SD, Talwar A, Lee JT. (2020) A proposed framework and timeline of the spectrum of disease due to SARS-CoV-2 infection: illness beyond acute infection and public health implications. JAMA 324(22):2251-2252.

5. Gupta A, Madhavan MV, Sehgal K, et al. (2020) Extrapulmonary manifestations of COVID-19. Nat Med 26:1017-10132.

6. Chen T, Wu D, Chen H, Yan W, Yang D, Chen G, et al. (2020) Clinical characteristics of 113 deceased patients with coronavirus disease 2019: retrospective study. BMJ.368:m1091.

7. Siripanthong B, Nazarian S, Muser D, et al. (2020) Recognizing COVID-19-related myocarditis: the possiblepathophysiology and proposed guideline for diagnosis and management. Heart Rhythm. S1547-5271(20)30422-7.

8. Huang X, Sun Y, Su G, Li Y, Shuai X. (2019) Intravenous immunoglobulin therapy for acute myocarditis in children and adults. Int Heart J. 60:359-365.

Ready to submit your research? Choose Auctores and benefit from:

* fast, convenient online submission

* rigorous peer review by experienced research in your field

* rapid publication on acceptance

* authors retain copyrights

* unique DOI for all articles

* immediate, unrestricted online access

At Auctores, research is always in progress.

Learn more www.auctoresonline.org/journals/cardiology-research-andreports 